\title{
Differential Cross-Sectional and Longitudinal Impact of APOE Genotype on Hippocampal Volumes in Nondemented Older Adults
}

\author{
Amy J. Jak ${ }^{a, b}$ Wes S. Houston ${ }^{c}$ Bonnie J. Nagel ${ }^{d}$ Jody Corey-Bloom ${ }^{b}$ e \\ Mark W. Bondi ${ }^{b, f}$ \\ aVeteran's Medical Research Foundation, and bVA San Diego Healthcare System, San Diego, Calif., 'Department of \\ Neurology, University of lowa, lowa City, lowa, 'Departments of Psychiatry and Behavioral Neuroscience, \\ Oregon Health and Science University, Portland, Oreg., and Departments of ${ }^{\mathrm{e}}$ Neurosciences and ${ }^{\mathrm{f}}$ Psychiatry, \\ University of California San Diego, San Diego, Calif., USA
}

\section{Key Words}

Apolipoprotein E $\varepsilon 4$ allele $\cdot$ Hippocampal volume $\cdot$

Cognitive performance

\begin{abstract}
Background/Aims: Because of conflicting findings across studies, we sought to better determine the relationship between apolipoprotein $\mathrm{E}$ (APOE) genotype, hippocampal volume, and cognitive performance in nondemented older adults. Methods: Two groups of older adults, as determined by their APOE $\varepsilon 4$ allele status, received structural MRI and comprehensive neuropsychological testing on two occasions separated on average by 17 months. Results: Crosssectional comparisons by APOE group revealed no differences in hippocampal volumes, although longitudinal percent reduction in hippocampal volume was significantly greater for those possessing the APOE $\varepsilon 4$ allele. Relationship between hippocampal volumes and memory performance was strongly impacted by diagnosis of mild cognitive impairment. Conclusions: APOE $\varepsilon 4$ allele appears to significantly impact rate of volume loss over time in the hippocampus in nondemented older adults, and detailed cognitive characterization of the sample is necessary to reliably interpret the relationship between cognition and brain structure.

Copyright $\odot 2007$ S. Karger AG, Basel
\end{abstract}

\section{Introduction}

Identifying early biomarkers of Alzheimer's disease (AD) allows for earlier opportunities to arrest the devastating neuropathological changes associated with $\mathrm{AD}$. One known risk factor for $\mathrm{AD}$ is the presence of the $\varepsilon 4$ allele of the apolipoprotein E gene (APOE). Hippocampal atrophy also has consistently been shown to accompany $\mathrm{AD}$ [1], with greater hippocampal atrophy noted in those with $\mathrm{AD}$ who possess a copy of the APOE $\varepsilon 4$ allele [2], but the literature remains clouded regarding whether APOE genotype is associated with hippocampal changes prior to disease onset.

Within cross-sectional designs, varied methodologies and cognitive status of study participants across studies has resulted in conflicting results on the effect of the APOE $\varepsilon 4$ allele on hippocampal volumes in nondemented older adults [3-10]. Studies that have more rigorously defined their samples as cognitively normal (i.e. no inclusion of mild memory decline or other abnormal cognition) have more consistently found that hippocampal volumes are comparable between APOE groups [7, 9]. However, some investigations of mild cognitive declines, such as in age-associated memory impairment, have also failed to find an impact of APOE on hippocampal volumes [11]. Longitudinal studies have been more consistent in find-

\section{KARGER}

Fax +4161306 1234 E-Mail karger@karger.ch www.karger.com
Mark W. Bondi, $\mathrm{PhD}$

University of California San Diego, Department of Psychiatry/

VA San Diego Healthcare System, 3350 La Jolla Village Drive (116B)

San Diego, CA 92161 (USA)

Tel. +1 858552 8585, ext. 2809, Fax +1 858642 1218, E-Mail mbondi@ucsd.edu 
ing relationships between changes in hippocampal volume and APOE genotype [7,8].

A similar debate exists regarding hippocampal asymmetry and APOE genotype. A 'normal' asymmetry is present in the hippocampus wherein right-sided volumes are consistently larger than left-sided volumes in normal adults [12], and left-greater-than-right discrepancies may be associated with pathology. Although some studies have found strong, even gene-dose-dependent, relationships of hippocampal asymmetry with APOE genotype in demented and nondemented samples [13, 14], others have failed to find altered asymmetry as a function of APOE genotype $[9,15]$.

The relationship of MRI-derived hippocampal volumes to neuropsychological performance on memory measures has also been somewhat equivocal. Several studies have reported larger hippocampal volumes are associated with better memory performance [16-19], though not all investigations have found this relationship [20]. Several studies, in fact, have found hippocampal volumes to be related to memory performance in cognitively impaired samples, but not in cognitively healthy participants [21-23] or more mildly impaired individuals, such as in age-associated memory impairment [24]. When evaluated longitudinally, some researchers have found hippocampal volume and memory decline to be related for those who progress to AD [25], though others have asserted that the role of APOE $\varepsilon 4$ genotype alone has not consistently predicted cognitive decline [26]. Often, APOE genotype has been associated with more rapid decline in memory functioning over time when it is also related to impaired baseline memory performance [27].

In spite of more than a decade of work in this area, the impact of APOE genotype on hippocampal volumes in nondemented older adults remains unclear. Therefore, we sought to better determine the degree to which APOE genotype impacts hippocampal volume. Utilizing high-resolution MR images and a highly reliable standardized manual outlining method, we examined hippocampal volumes in a neuropsychologically welldefined, nondemented sample of elders. Due to the limited but consistent longitudinal findings, we hypothesized that, when analyzed cross-sectionally, APOE genotype would not significantly impact hippocampal volumes in this group. When analyzed longitudinally, however, we predicted that those positive for the APOE $\varepsilon 4$ allele would exhibit greater decline in hippocampal volumes over time.

Impact of APOE Genotype on

Hippocampal Volumes
Table 1. Participant diagnoses by APOE genotype group

\begin{tabular}{lclll}
\hline & Normal & $\begin{array}{l}\text { MCI amnestic } \\
\text { single domain }\end{array}$ & $\begin{array}{l}\text { MCI amnestic } \\
\text { multi-domain }\end{array}$ & $\begin{array}{l}\text { Nonam- } \\
\text { nestic }\end{array}$ \\
\hline Cross-sectionally & & & & \\
APOE $\varepsilon 4$ & 16 & 3 & 2 & 1 \\
APOE non- 4 4 & 36 & 2 & 4 & 5 \\
Total & 52 & 5 & 6 & 6 \\
\hline Longitudinally & & & & \\
APOE $\varepsilon 4$ & 8 & 1 & 1 & 0 \\
APOE non- 44 & 26 & 0 & 1 & 2 \\
Total & 34 & 1 & 2 & 2 \\
\hline
\end{tabular}

\section{Methods}

\section{Participants}

The appropriate institutional review boards approved this study and all participants provided written informed consent. Sixty-nine medically healthy, nondemented, and independently living participants, ages 63-92, were studied. This was a volunteer cohort, recruited solely for research purposes, drawn from a larger pool of 110 individuals enrolled in a longitudinal study of aging. Participants were consecutively accrued and selected because they had undergone both an MRI and neuropsychological evaluation. Additionally, all participants received medical, neurological, and laboratory evaluations. Those with a history of alcoholism, drug abuse, learning disability, neurological, or psychiatric illness (including depression) were excluded. All participants were right-handed. Thirty-nine of these participants had followup imaging and neuropsychological data that were utilized in longitudinal analyses. Average span between study visits was 17 months (standard deviation, $\mathrm{SD}=5.3$ months). There were no significant genotype $\left(\chi^{2}=2.20, p=0.14\right)$, diagnostic $\left(\chi^{2}=1.81\right.$, $\mathrm{p}=0.18$ ), demographic, or cognitive (all $\mathrm{p}$ values $>0.14$ ) differences between those who were available for follow-up compared to those who were not.

The comprehensive neuropsychological battery included measures of global intellectual and cognitive functioning (e.g. Dementia Rating Scale (DRS) [28], language), attention, executive functioning, and memory. Tests of memory (i.e. DRS Memory Subscale; Wechsler Memory Scale-Revised [29] Logical Memory (WMS-R LM) subtest, and California Verbal Learning Test (CVLT) [30]), were the focus of the present investigation as medial temporal lobe structures mediate episodic memory and are strong indicators of future decline [31]. To objectively determine the presence or absence of functional impairment, we also utilized the Independent Living Scales (ILS) [32], an ecologically valid measure designed to assess one's ability to complete independent activities of daily living.

Following completion of all evaluations, a consensus committee of two neuropsychologists and a neurologist was assembled to determine if any participants met criteria for a cognitive disorder. Subjects with, at most, one score falling 1 SD or more below their age-appropriate norms on the comprehensive neuropsychological assessment [for discussion, see 33], in the absence of functional impairment, were characterized as neuropsychologically normal. 
Table 2. Cross-sectional demographic, global cognitive, and learning and memory characteristics by APOE genotype group (mean $\pm \mathrm{SD}$ )
Table 3. Follow-up demographic, global cognitive, and learning and memory characteristics by APOE genotype group (mean $\pm \mathrm{SD})$

\begin{tabular}{lcclc}
\hline Variable & $\begin{array}{c}\text { Non- } \varepsilon 4 \\
(\mathrm{n}=47)\end{array}$ & $\begin{array}{l}\varepsilon 4 \\
(\mathrm{n}=22)\end{array}$ & $\mathrm{t}$ & $\mathrm{p}$ \\
\hline Age & $77.6 \pm 6.7$ & $74.9 \pm 7.2$ & 1.5 & 0.13 \\
Years of education & $16.1 \pm 2.5$ & $15.6 \pm 2.2$ & 0.71 & 0.48 \\
Gender (women/men) & $26 / 21$ & $10 / 12$ & $\chi^{2}=0.58$ & 0.44 \\
ANART VIQ & $119.1 \pm 6.4$ & $119.3 \pm 6.3$ & 0.13 & 0.90 \\
DRS Total T score & $53.2 \pm 5.7$ & $53.1 \pm 4.4$ & 0.07 & 0.95 \\
DRS Memory T score & $51.9 \pm 5.7$ & $51.5 \pm 9.7$ & 0.17 & 0.86 \\
WMS-R LM Immediate recall scaled score & $12.3 \pm 3.1$ & $11.9 \pm 3.7$ & 0.46 & 0.65 \\
WMS-R LM Delayed recall scaled score & $12.5 \pm 3.0$ & $11.8 \pm 4.1$ & 0.82 & 0.42 \\
CVLT List 1-5 total recall T score & $52.5 \pm 11.1$ & $50.6 \pm 13.8$ & 0.62 & 0.53 \\
CVLT Long delay free recall T score & $48.2 \pm 12.9$ & $49.7 \pm 15.3$ & 0.42 & 0.68 \\
ILS Managing money T score & $56.22 \pm 5.0$ & $54.24 \pm 4.7$ & 0.01 & 0.91 \\
ILS Health and safety T score & $55.0 \pm 6.4$ & $55.3 \pm 4.7$ & 2.04 & 0.16 \\
\hline
\end{tabular}

ANART VIQ = American National Adult Reading Test, Verbal IQ.

\begin{tabular}{lccll}
\hline Variable & $\begin{array}{l}\text { Non- } \varepsilon 4 \\
(\mathrm{n}=29)\end{array}$ & $\begin{array}{l}\varepsilon 4 \\
(\mathrm{n}=10)\end{array}$ & $\mathrm{t}$ & $\mathrm{p}$ \\
\hline Age & $77.5 \pm 6.3$ & $75.1 \pm 5.9$ & 1.1 & 0.30 \\
Years of education & $16.3 \pm 2.4$ & $15.9 \pm 2.6$ & 0.45 & 0.66 \\
Gender (women/men) & $15 / 14$ & $5 / 5$ & $\chi^{2}=0.01$ & 0.93 \\
ANART VIQ & $118.6 \pm 7.0$ & $120.2 \pm 6.0$ & 0.65 & 0.52 \\
DRS Total T score & $54.4 \pm 3.7$ & $51.7 \pm 10.4$ & 1.17 & 0.25 \\
DRS Memory T score & $52.7 \pm 7.7$ & $49.7 \pm 9.8$ & 0.95 & 0.35 \\
WMS-R LM Immediate recall scaled score & $12.9 \pm 2.8$ & $11.1 \pm 3.3$ & 1.45 & 0.16 \\
WMS-R LM Delayed recall scaled score & $13.1 \pm 3.0$ & $11.6 \pm 3.6$ & 1.14 & 0.27 \\
CVLT List 1-5 total recall T score & $54.7 \pm 11.8$ & $50.9 \pm 11.9$ & 0.85 & 0.40 \\
CVLT Long delay free recall T score & $47.0 \pm 11.1$ & $43.4 \pm 15.9$ & 0.70 & 0.49 \\
ILS Managing money T score & $58.3 \pm 3.6$ & $57.0 \pm 5.2$ & 0.58 & 0.57 \\
ILS Health and safety T score & $59.3 \pm 5.2$ & $60.5 \pm 5.0$ & -0.42 & 0.68 \\
\hline
\end{tabular}

In order to strike a balance between diagnostic validity and sensitivity to detect mild impairment, subjects were defined as mildly cognitively impaired (MCI) based on criteria suggested by $\mathrm{Pe}$ tersen and Morris [34], including absence of dementia and functional impairment. Amnestic and nonamnestic MCI subtypes were included. Impairment was operationally defined as performance on standardized neuropsychological tests of $>1$ SD below age-appropriate norms on at least two measures within a cognitive domain (single domain amnestic: impaired memory; multiple domain amnestic: impaired memory and impaired attention, language, visuospatial processing, executive functioning, and/or processing speed; nonamnestic: impairment in one or more nonmemory domain). In sum, consensus diagnoses revealed that, initially, 52 participants were characterized as cognitively normal and 17 participants were characterized as MCI. In the longitudinal follow-up, 34 participants were characterized as cognitively normal and 5 were MCI (table 1).

All participants were genotyped for APOE allele type using a polymerase chain reaction-based method. Two demographically equated groups were established based on the presence or absence of the APOE $\varepsilon 4$ allele. The initial $\varepsilon 4$ group comprised 22 individuals who had a least one copy of the APOE $\varepsilon 4$ allele and the non- $\varepsilon 4$ group had 47 participants who did not possess any copies of the APOE $\varepsilon 4$ allele. At follow-up, the $\varepsilon 4$ group comprised 10 individuals and the non- $\varepsilon 4$ group had 29 participants.

In the initial full cross-sectional sample (table 2) and the longitudinal cohort (table 3), the APOE groups did not differ on demographic characteristics, global cognition (DRS) or memory functioning (WMS-R LM or CVLT; all p values $>0.05$ ). Furthermore, the APOE groups did not differ on demographic characteristics or neuropsychological performance when the individuals with MCI were removed from the sample (all $p$ values $>0.05$ ). Not surprisingly, MCI participants performed significantly more poorly on memory measures than did the cognitively normal group; however, the diagnostic groups did not differ on age, education, gender, or ADL variables (table 4). It is also of note that the MCI participants were evenly distributed between the APOE groups in both the cross-sectional $\left(\chi^{2}=1.21\right.$, $\mathrm{p}=0.73)$ and longitudinal $\left(\chi^{2}=0.62, \mathrm{p}=0.43\right)$ samples. 
Table 4. Cross-sectional demographic, global cognitive, and learning and memory characteristics by diagnostic group (mean $\pm \mathrm{SD})$

\begin{tabular}{lcccc}
\hline Variable & $\begin{array}{l}\text { Normal } \\
(\mathrm{n}=52)\end{array}$ & $\begin{array}{l}\text { MCI } \\
(\mathrm{n}=17)\end{array}$ & $\mathrm{t}$ & $\mathrm{p}$ \\
\hline Age & $76.6 \pm 7.0$ & $77.3 \pm 6.7$ & 0.37 & 0.71 \\
Years of education & $15.9 \pm 2.3$ & $16.1 \pm 2.8$ & 0.23 & 0.82 \\
Gender (women/men) & $30 / 22$ & $6 / 11$ & $\chi^{2}=2.58$ & 0.11 \\
ANART VIQ & $120.0 \pm 6.1$ & $116.3 \pm 6.1$ & 2.11 & 0.04 \\
DRS Total T score & $55.1 \pm 4.0$ & $47.3 \pm 8.1$ & 3.80 & 0.001 \\
DRS Memory T score & $54.4 \pm 4.3$ & $43.7 \pm 12.8$ & 3.37 & 0.004 \\
WMS-R LM Immediate recall scaled score & $13.2 \pm 2.4$ & $8.8 \pm 3.3$ & 5.19 & 0.000 \\
WMS-R LM Delayed recall scaled score & $13.5 \pm 2.4$ & $8.7 \pm 3.3$ & 5.55 & 0.000 \\
CVLT List 1-5 total recall T score & $55.0 \pm 10.4$ & $42.2 \pm 11.3$ & 4.32 & 0.000 \\
CVLT Long delay free recall T score & $52.4 \pm 11.3$ & $37.2 \pm 14.2$ & 4.52 & 0.000 \\
ILS Managing money T score & $56.4 \pm 4.5$ & $53.6 \pm 5.5$ & 1.95 & 0.06 \\
ILS Health and safety T score & $55.5 \pm 6.3$ & $53.9 \pm 4.7$ & 0.88 & 0.38 \\
\hline
\end{tabular}

Imaging, Regions of Interest Protocol, and Segmentation

High-resolution $\mathrm{T}_{1}$-weighted whole-brain anatomical MR images were obtained using a 1.5-Tesla GE Signa imager utilizing an SPGR sequence (124 slices acquired in the sagittal plane; $1.2 \mathrm{~mm}$ slice thickness; $256 \times 256$ matrix; FOV $=250 \mathrm{~mm}$; resulting in a 1 $\mathrm{mm}^{2}$ in-plane resolution). Hippocampal volumes were obtained (bilaterally) via visual inspection and manual outlining performed in the coronal plane. Images were realigned perpendicular to the anterior-posterior commissure line, but not transformed into standard space coordinates. Regions of interest were delineated using Analysis of Functional NeuroImages software and completed by an experienced operator (A.J.J.), who was blind to participant identity and group. High levels of intra- and inter-rater reliability for the procedure were established on a separate set of images not among those studied presently (intraclass correlation coefficients $>0.90$ ). Hippocampal regions of interest were delineated using a stereotactic approach adapted from methods published previously [35]. The anterior bound of the hippocampus was chosen as the coronal slice through the fullest portion of the mammillary bodies, and the posterior boundary was traced on the last coronal slice on which the superior colliculi could be fully visualized. Whole-brain images were also skull-stripped and segmented into gray matter, white matter, and cerebrospinal fluid (CSF) compartments. Scans were manually edited when necessary to remove any residual nonbrain material. Whole-brain volume was derived and used in normalizing hippocampal, total gray matter, white matter, and CSF volumes [36].

\section{Results}

\section{Whole-Brain and Hippocampal Volumes}

At the initial visit, MANCOVA controlling for wholebrain volume revealed neither a main multivariate effect $\left(\mathrm{F}=0.55, \mathrm{p}=0.65, \eta_{\mathrm{p}}{ }^{2}=0.03\right)$ nor significant univariate effects of APOE group for normalized tissue segmentation volumes (gray: $\mathrm{F}=0.45, \mathrm{p}=0.50, \eta_{\mathrm{p}}{ }^{2}=0.01$; white: $\mathrm{F}=0.85, \mathrm{p}=0.36, \eta_{\mathrm{p}}{ }^{2}=0.01 ; \mathrm{CSF}: \mathrm{F}=0.52 \mathrm{p}=0.47, \eta_{\mathrm{p}}{ }^{2}=$ $0.01)$. Regarding the analysis of the hippocampus at time
1 , results revealed no main multivariate effect for APOE genotype $\left(\mathrm{F}=1.45, \mathrm{p}=0.24, \eta_{\mathrm{p}}{ }^{2}=0.04\right)$ and no main univariate effect of group for either right- or left-sided volumes (right: $F=0.24, p=0.62, \eta_{p}{ }^{2}=0.004$; left: $F=0.23$, $\left.\mathrm{p}=0.64, \eta_{\mathrm{p}}^{2}=0.003\right)$. When those with MCI were removed from the sample and analyses were repeated, hippocampal differences between APOE groups still did not emerge ( $p$ values $>0.13$ ). When examining the impact of diagnosis, results revealed no main multivariate or univariate effects for diagnostic group for either right or left hippocampal volumes, or whole-brain, gray, white, or CSF volumes ( $\mathrm{p}$ values $>0.12$ ).

No multivariate $\left(\mathrm{F}=0.26, \mathrm{p}=0.85, \eta_{\mathrm{p}}^{2}=0.02\right)$ or univariate (gray: $\mathrm{F}=0.30, \mathrm{p}=0.59, \eta_{\mathrm{p}}{ }^{2}=0.01$; white: $\mathrm{F}=0.08$, $\mathrm{p}=0.78, \eta_{\mathrm{p}}{ }^{2}=0.002 ;$ CSF: $\left.\mathrm{F}=0.15, \mathrm{p}=0.70, \eta_{\mathrm{p}}{ }^{2}=0.004\right)$ differences were found at follow-up for normalized tissue segmentation volumes. Analyses of the hippocampus at time 2 revealed a main multivariate effect for APOE genotype $\left(\mathrm{F}=3.35, \mathrm{p}=0.05, \eta_{\mathrm{p}}{ }^{2}=0.16\right)$ but no main univariate effect of group for either right- or left-sided volumes (right: $F=0.16, p=0.69, \eta_{p}{ }^{2}=0.005$; left: $F=3.26$, $\left.\mathrm{p}=0.08, \eta_{\mathrm{p}}{ }^{2}=0.08\right)$. When those with MCI were removed from the sample and analyses were repeated, multivariate and univariate hippocampal differences between APOE were eliminated ( $\mathrm{p}$ values $>0.23$ ).

Longitudinal change in bilateral hippocampal volumes revealed that percent change bilaterally was greater in those positive for the APOE $\varepsilon 4$ allele than in those negative for the allele. Specifically, in the $\varepsilon 4$ group, rightsided volumes decreased by $9.1 \%$ while in the non- $\varepsilon 4$ group the volume decreased at a reduced rate of $3.0 \%(t=$ $1.6, \mathrm{p}=0.12)$. On the left, the volume of the $\varepsilon 4$ group was decreased by $11.4 \%$, while the non- $\varepsilon 4$ group left-sided volume decreased at a significantly reduced rate of $1.5 \%$ 


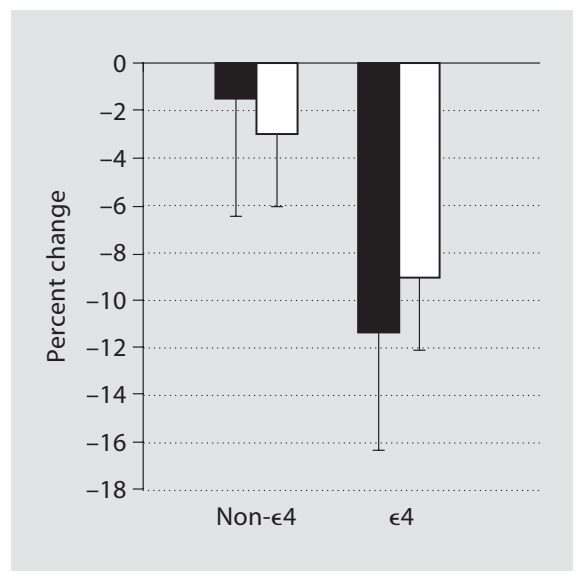

Fig. 1. Percent change in bilateral hippocampal volumes. $\mathbf{\square}=$ Left; $\square=$ right.

$(\mathrm{t}=2.9, \mathrm{p}=0.01$; fig. 1$)$. Examining the individual trajectories of the participants suggests less variability and more consistent volumetric declines among the $\varepsilon 4$ group (fig. 2). There were no significant differences by APOE genotype on percent change in whole-brain volume $(t=$ $0.55, \mathrm{p}=0.58$ ). Removing those with MCI diagnoses did not significantly alter results.

\section{Hippocampal Asymmetry}

To address hippocampal asymmetry, a repeated-measures analysis of variance was conducted, with APOE group as the between-group factor and hemisphere (right vs. left hippocampus) as the within-subjects repeated measure. At time 1, as expected, there was a main effect of hemisphere $\left[\mathrm{F}(2,67)=31.37, \mathrm{p}<0.001, \eta_{\mathrm{p}}{ }^{2}=0.32\right]$, indicating that right-sided volumes were larger than left in both groups. The interaction of group and hemisphere was only marginally significant $\left(F=2.92, p=0.09, \eta_{p}^{2}=0.04\right)$, indicating that the rightward asymmetry was larger for the non- $\varepsilon 4$ group (fig. 3). Removal of individuals with MCI from the analyses did not significantly alter results.

At time 2, a main effect of hemisphere $[\mathrm{F}(2,37)=8.77$, $\mathrm{p}<0.01]$ emerged and the interaction of group and hemisphere approached significance $(\mathrm{F}=3.80, \mathrm{p}=0.06)$, suggesting that the rightward asymmetry was larger for the non- $\varepsilon 4$ group. Excluding MCI participants from the follow-up sample resulted in a main effect of hemisphere $[\mathrm{F}(2,34)=7.61, \mathrm{p}<0.01]$, thought the interaction of group and hemisphere was not statistically significant $(\mathrm{F}=2.44$, $\mathrm{p}=0.13)$.

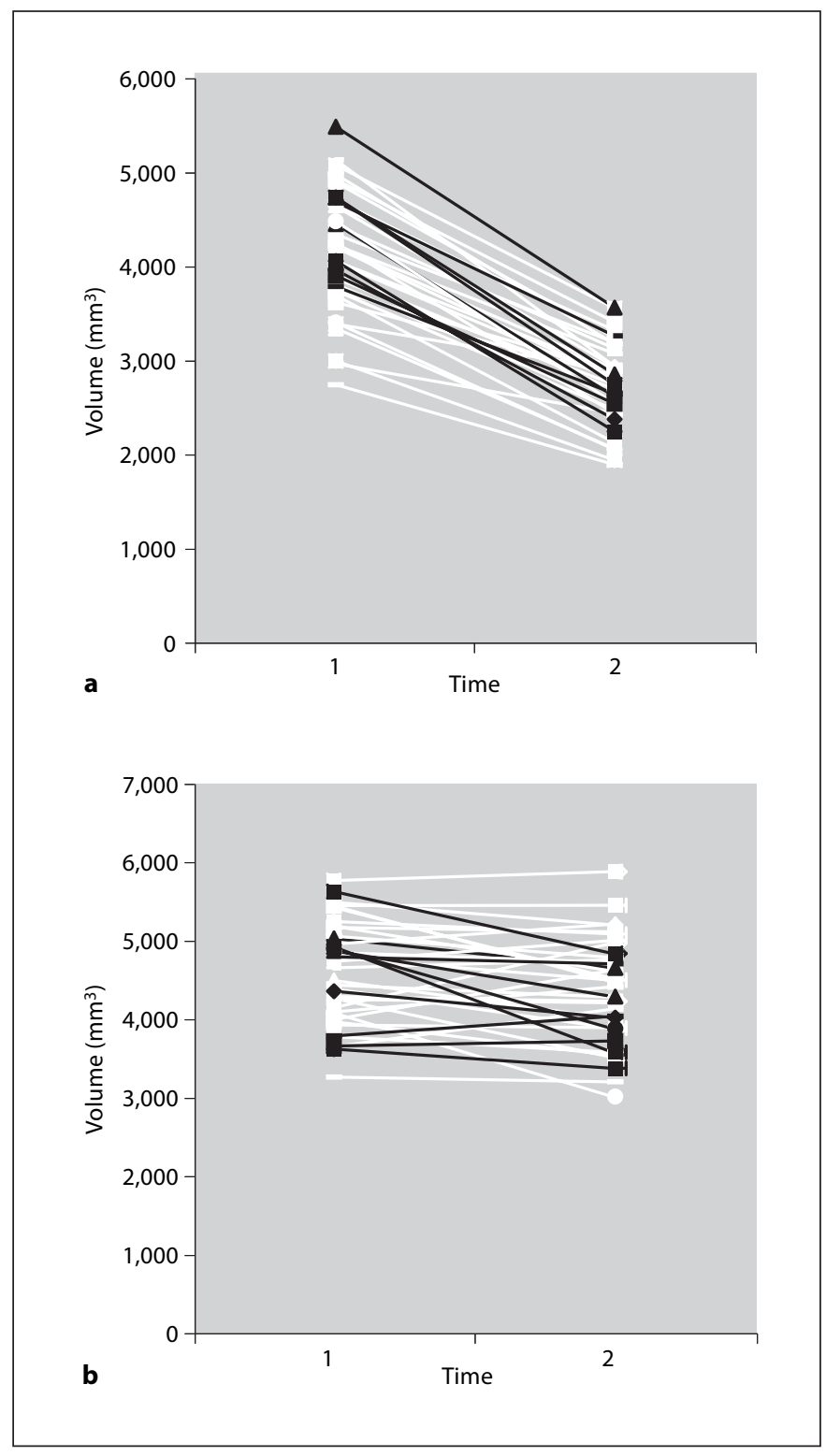

Fig. 2. Left (a) and right (b) hippocampal volumes by subject. White lines $=$ Non- $\varepsilon 4$; black lines $=\varepsilon 4$.

\section{APOE-Hippocampal-Memory Associations}

Due to reduced sample sizes, particularly within certain cells once those with MCI were removed for certain analyses, nonparametric correlations were conducted. At the initial visit, larger left normalized hippocampal volumes were associated with better performance on the ILS Health and Safety subscale among those negative for the APOE $\varepsilon 4$ allele (Spearman's rho $=0.34 ; \mathrm{p}=0.04$ ). However, there was no significant relationship between left normalized hippocampal volumes and ILS Health and 


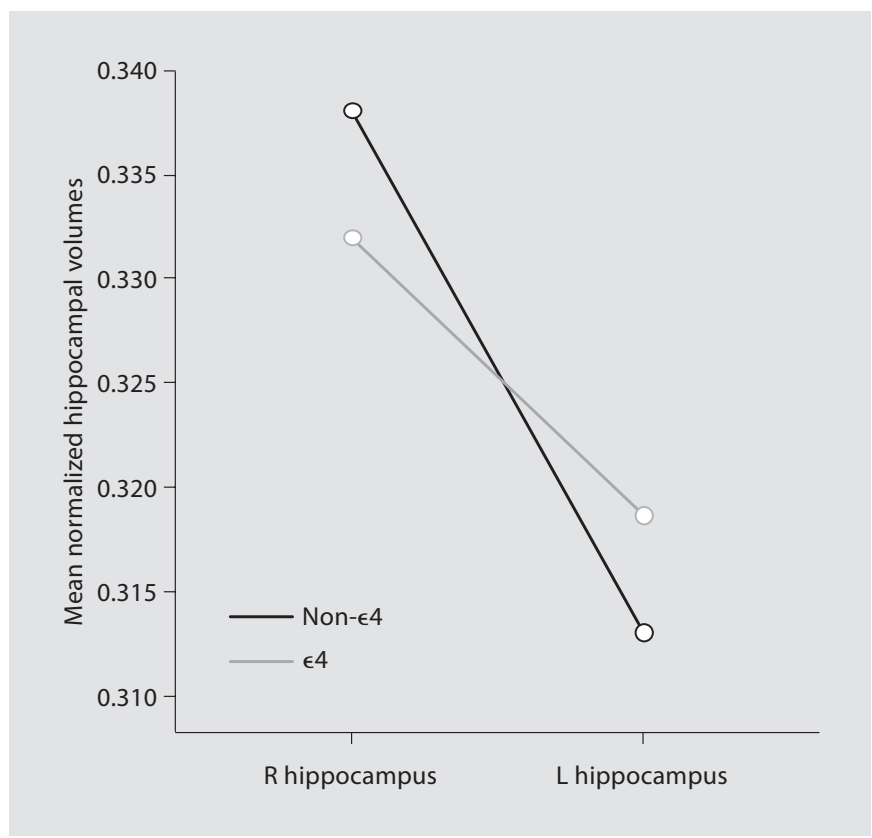

Fig. 3. Hippocampal asymmetry by APOE group.

safety in those positive for the $\varepsilon 4$ allele (Spearman's rho = $0.08 ; \mathrm{p}=0.76$ ). Removing those with MCI from the sample did not significantly alter results. At follow-up, this positive relationship remained. A trend was found toward an association between larger left normalized hippocampal volumes and better performance on the ILS Health and safety subscale among those negative for the APOE $\varepsilon 4$ allele (Spearman's rho $=0.52 ; \mathrm{p}=0.09$ ) as well as in those positive for the $\varepsilon 4$ allele (Spearman's rho $=$ $0.78 ; \mathrm{p}=0.23$ ). Removing those with MCI from the sample did not significantly alter results.

Regarding memory, at the initial visit, no association emerged between left hippocampal volumes and performance on WMS-R LM Immediate or Delayed recall in either genotype group, irrespective of inclusion or exclusion of individuals with MCI (all $p$ values $>0.21$ ). At the follow-up visit, larger left normalized hippocampal volumes were associated with better WMS-R LM Immediate recall (Spearman's rho $=0.41 ; \mathrm{p}=0.03$ ) and Delayed recall (Spearman's rho $=0.41 ; \mathrm{p}=0.03$ ) among those negative for the APOE $\varepsilon 4$ allele. Similar trends emerged for those positive for the $\varepsilon 4$ allele (Immediate recall: Spearman's rho $=0.72 ; \mathrm{p}=0.07$; Delayed recall: Spearman's rho $=$ $0.72 ; \mathrm{p}=0.07)$. However, when those with MCI were removed from the follow-up sample, no significant relationships between left and hippocampal volumes and memory variables emerged in either genotype group.

\section{Discussion}

In this well-characterized, nondemented sample of older adults, APOE $\varepsilon 4$ allele status does not significantly impact cross-sectional between-group variation in hippocampal volumes. However, APOE genotype is related to hippocampal volumetric declines over time. Both $\mathrm{APOE} \varepsilon 4$ groups showed the 'normal' right-greater-thanleft hippocampal asymmetry at initial and follow-up visits, although this expected asymmetry was less robust in the group positive for the APOE $\varepsilon 4$ allele. The relationship between memory and hippocampal volume was largely a function of the cognitive characterization of the sample. That is, excluding individuals with MCI tended to eliminate any relationship between brain structure and memory.

Regarding approaches to hippocampal volumetric measurements in the healthy elderly, our results stand in contrast to several prior studies that have found smaller hippocampal volumes in those who possess the APOE $\varepsilon 4$ allele [3-5]. Methodological variability between studies may be a contributing factor to some of these discrepancies [3,37]. Perhaps more important are differences across studies in fully characterizing the cognitive composition of participant samples. Some studies appeared to use only the Mini-Mental State Exam to cognitively characterize their groups [3], which often lacks consistency in accurately describing the full range of cognitive performance [31]. Specifically, Salmon et al. [38] point out that the traditional Mini-Mental State Exam cutoff score of 24 and below for dementia is too conservative and showed that persons with scores greater than 24 can also be accurately diagnosed with AD. Further, some studies did not provide sufficient information about cognitive functioning of the sample or group compositions. This study attempted to more thoroughly document the makeup of our participant sample and to examine the impact of inclusion of MCI participants.

Our results indicate that neither a neuropsychologically normal sample nor a sample of mixed neuropsychologically normal and MCI individuals demonstrate differences in hippocampal volumes as a function of $\mathrm{APOE}$ status when examined cross-sectionally. Despite including impaired (though not demented) individuals in an otherwise normal sample, it is possible that hippocampal volumetric differences and altered hippocampal asymmetry did not emerge between genotype groups because the MCI participants were evenly distributed between the genotype groups, effectively canceling each other out. Other studies demonstrating hippocampal differences 
may have had more MCI individuals overrepresented within their APOE $\varepsilon 4$ groups. Our failure to find hippocampal differences or altered asymmetry between APOE $\varepsilon 4$ groups is more consistent with other studies that have also more comprehensively characterized the cognitive presentation of their cross-sectional sample $[7,9]$. The literature generally suggests that, once cognitive decline is manifest, hippocampal volumetric differences by APOE genotype are more readily identified if subjects are not well equated between APOE groups. In these cases, the structural correlate of these cognitive differences, and not structural change associated with APOE genotype independent of cognitive decline, is likely what is measured. While these results add some clarity to the conflicting literature in this area, the study is limited by the relatively small $\varepsilon 4$ sample, and replication with larger sample sizes is warranted.

Our finding of greater volumetric declines over time in the hippocampus in the APOE $\varepsilon 4$ group is consistent with the limited number of longitudinal studies in this area [7, 8]. Diagnostic status did not impact the longitudinal analyses possibly due to the fact that each participant serves as her/his own control. Several participants' diagnostic characterization changed over time and APOE genotype status remained related to percent change over time in hippocampal volumes. Perhaps the presence of the APOE $\varepsilon 4$ allele exerts its impact via an accelerated atrophy of the hippocampus, even in nondemented individuals, a difference that is not reliably detected when examining samples cross-sectionally. Additionally, the trend towards a difference in the degree of right-left hippocampal asymmetry between genotype groups may suggest that a manifestation of APOE genotypic risk is exerted in a reduction in normal hippocampal asymmetry.

Our results highlight that diagnostic state is integral in determining the association between hippocampal volume, genotypic risk, and memory. The relationship between volume, genotype, and memory was present when those with MCI were included, but not when studying a fully cognitively normal sample. Removal of MCI subjects from the analyses is a limitation in that it reduced statistical power. However, visual inspection of the data plots of these correlations suggests that the relationships are not spurious. Sample size of the MCI participants precludes commenting definitively about characteristics of this particular subset (a sample size of 50 would be required to reliably compare the MCI and nonimpaired groups with a power of 0.80 ). However, the altered results obtained upon their removal from the sample highlight the need for careful cognitive characteriza- tion of samples of older adults. This caution is also well aligned with other studies that have found that associations between hippocampal volumes and memory are predominantly due to inclusion of more cognitively impaired groups, such as those with $\mathrm{AD}$ [23].

It is also of interest that a more robust relationship between structure and function emerged when examining more ecologically valid tests of functioning (i.e. ILS), a relationship that persisted irrespective of diagnosis. However, the relationship between structure and memory function was strongly influenced by cognitive diagnosis.

It is possible that the relationship between hippocampal volume and functional status persists regardless of diagnosis in the present study as all participants, even those with MCI, had to be free of functional impairments. In those without the APOE $\varepsilon 4$ allele, poorer (though not impaired) objective functional status relates to smaller hippocampal volumes. This relationship should be further explored to determine if utilizing structure and function combinations, particularly in those without genetic liability for $\mathrm{AD}$, can be used to improve prediction of those at highest risk for dementia.

In summary, when examined cross-sectionally, APOE genotype does not appear to impact hippocampal volume in nondemented individuals but it does exert influence in rate of atrophy over time. Furthermore, in our aging sample, APOE genotype does not affect the presence of the predicted hippocampal asymmetry, although it does influence the robustness of this asymmetry in those with the $\varepsilon 4$ allele. As such, longitudinal decline in hippocampal volumes as well as hippocampal asymmetry may hold more promise as possible biomarkers of an approaching dementia than absolute differences within either the left or right hippocampus examined at only one time point. Hippocampal volume and APOE status are related to memory functioning, but only within a mixed sample including both neuropsychologically normal and MCI individuals, a finding that speaks to the importance of thorough cognitive characterization of elderly samples to avoid spurious results due to any overrepresentation of MCI among those positive for the APOE $\varepsilon 4$ allele.

\section{Acknowledgments}

This work was supported by National Institute on Aging grants R01 AG12674 and P50 AG05131. The authors gratefully acknowledge the assistance of staff, patients, and volunteers of the UCSD Alzheimer's Disease Research Center, and the UCSD Laboratory of Cognitive Imaging. 


\section{References}

1 Poulin P, Zakzanis KK: In vivo neuroanatomy of Alzheimer's disease: evidence from structural and functional brain imaging. Brain Cogn 2002;49:220-225.

$\checkmark 2$ Geroldi C, Pihlajamaki M, Laakso MP, et al: APOE-epsilon 4 is associated with less frontal and more medial temporal lobe atrophy in AD. Neurology 1999;53:1825-1832.

$\checkmark 3$ Tohgi H, Takahashi S, Kato E, et al: Reduced size of right hippocampus in 39- to 80-yearold normal subjects carrying the apolipoprotein E epsilon 4 allele. Neurosci Lett 1997; 236:21-24.

$\checkmark 4$ den Heijer T, Oudkerk M, Launer LJ, van Duijn CM, Hofman A, Breteler MMB: Hippocampal, amygdalar, and global brain atrophy in different apolipoprotein E genotypes. Neurology 2002;59:746-748.

5 Plassman BL, Welsh-Bohmer KA, Bigler ED, et al: Apolipoprotein E epsilon 4 allele and hippocampal volume in twins with normal cognition. Neurology 1997;48:985-989.

6 Jernigan TL, Archibald SL, Fennema-Notestine C, et al: Effects of age on tissues and regions of the cerebrum and cerebellum. Neurobiol Aging 2001;22:581-894.

$\checkmark 7$ Cohen RM, Small C, Lalonde F, Friz J, Sunderland T: Effect of apolipoprotein E genotype on hippocampal volume loss in aging healthy women. Neurology 2001;57:22232228.

8 Moffat SD, Szekely CA, Zonderman AB, Kabani NJ, Resnick SM: Longitudinal change in hippocampal volume as a function of apolipoprotein E genotype. Neurology 2000;55: 134-136.

-9 Reiman EM, Ueker A, Caselli RJ, et al: Hippocampal volumes in cognitively normal persons at genetic risk for Alzheimer's disease. Ann Neurol 1998;44:288-291.

10 Tupler LA, Krishnan KRR, Greenberg DL, et al: Predicting memory decline in normal elderly: genetics, MRI, and cognitive reserve. Neurobiol Aging, Epub ahead of print.

- 11 Serra-Grabulosa JM, Salgado-Pineda P, Junque C, et al: Apolipoproteins E and C1 and brain morphology in memory impaired elders. Neurogenetics 2003;4:141-146.

-12 Pedraza O, Bowers D, Gilmore R: Asymmetry of the hippocampus and amygdala in MRI volumetric measurements of normal adults. J Int Neuropsychol Soc 2004;10:664678 .
13 Soininen H, Partanen K, Pitkanen A, et al: Decreased hippocampal volume asymmetry on MRIs in nondemented elderly subjects carrying the apolipoprotein E epsilon4 allele. Neurology 1995;45:391-392.

14 Geroldi C, Laakso MP, DeCarli C, et al: Apolipoprotein $\mathrm{E}$ genotype and hippocampal asymmetry in Alzheimer's disease: a volumetric MRI study. J Neurol Neurosurg Psychiatry 2000;68:93-96.

15 Bigler ED, Tate DF, Miller MJ, et al: Dementia, asymmetry of temporal lobe structures, and apolipoprotein E genotype: relationships to cerebral atrophy and neuropsychological impairment. J Int Neuropsychol Soc 2002;8:925-933

16 Lye TC, Piguet O, Grayson DA, et al: Hippocampal size and memory function in the ninth and tenth decades of life: the Sydney Older Persons Study. J Neurol Neurosurg Psychiatry 2004;75:548-554.

17 Hackert VH, den Heijer T, Oudkerk M, Koudstaal PJ, Hofman A, Breteler MMB: Hippocampal head size associated with verbal memory performance in nondemented elderly. Neuroimage 2002;17:1365-1372.

18 Walhovd KB, Fjell AM, Reinvang I, et al: Size does matter in the long run: hippocampal and cortical volume predict recall across weeks. Neurology 2004;63:1193-1197.

19 Mortimer JA, Gosche KM, Riley KP, Markesbery WR, Snowdon DA: Delayed recall, hippocampal volume and Alzheimer neuropathology. Neurology 2004;62:428-432.

20 Ylikoski R, Salonen O, Mantyla R, et al: Hippocampal and temporal lobe atrophy and age-related decline in memory. Acta Neurol Scand 2000;101:273-278.

21 Basso M, Yang J, Warren L, et al: Volumetry of amygdala and hippocampus and memory performance in Alzheimer's disease. Psychiatry Res 2006;146:251-261.

22 Persson J, Nyberg L, Lind J, et al: Structurefunction correlates of cognitive decline in aging. Cereb Cortex 2006;16:907-915.

23 Petersen RC, Jack CR, Xu Y-C, et al: Memory and MRI-based hippocampal volumes in aging and AD. Neurology 2000;54:581-587.

24 Bartres-Faz D, Junque C, Clemente IC, et al: MRI and genetic correlates of cognitive function in elders with memory impairment. Neurobiol Aging 2001;22:449-459.

$\checkmark 25$ Mungas D, Harvey D, Reed BR, et al: Longitudinal volumetric MRI change and rate of cognitive decline. Neurology 2005;65:565571.
26 Devanand DP, Pelton GH, Zamora D, et al: Predictive utility of apolipoprotein E genotype for Alzheimer disease in outpatients with mild cognitive impairment. Arch Neurol 2005;62:975-980.

27 Wilson RS, Schneider JA, Barnes LL, et al: The apolipoprotein E epsilon 4 allele and decline in different cognitive systems during a 6-year period. Arch Neurol 2002;59:11541160.

28 Mattis S: Dementia Rating Scale: Professional Manual. Odessa, Psychological Assessment Resources, 1988.

29 Wechsler D: Wechsler Memory Scale - Revised. New York, Psychological Corporation, 1987.

30 Delis DC, Kramer JH, Kaplan E, Ober BA: The California Verbal Learning Test. New York, Psychological Corporation, 1987.

-31 Twamley EW, Ropacki SAL, Bondi MW: Neuropsychological and neuroimaging changes in preclinical Alzheimer's disease. J Int Neuropsychol Soc 2006;12:707-735.

32 Loeb PA: ILS: Independent Living Scales Manual. San Antonio, Psychological Corp, Harcourt Brace Jovanovich, 1996.

33 Heaton RK, Grant I, Matthews CG: Comprehensive Norms for an Expanded HalsteadReitan Battery: Demographic Corrections, Research Findings, and Clinical Applications. Odessa, Psychological Assessment Resources, 1991.

34 Petersen RC, Morris JC: Mild cognitive impairment as a clinical entity and treatment target. Arch Neurol 2005;62:1160-1163.

35 Nagel BJ, Palmer SL, Reddick WE, et al: Abnormal hippocampal development in children with medulloblastoma treated with risk-adapted irradiation Am J Neuroradiol 2004;25:1575-1582.

36 Bigler ED, Tate DF: Brain volume, intracranial volume, and dementia. Invest Radiol 2001;36:539-546.

37 Geuze E, Vermetten E, Bremmer JD: MRbased in vivo hippocampal volumetrics. 1. Review of methodologies currently employed. Mol Psychiatry 2005;10:147-159.

38 Salmon DP, Thomas RG, Pay MM, et al: Alzheimer's disease can be accurately diagnosed in very mildly impaired individuals. Neurology 2002;59:1022-1028. 